\title{
Remote Site Monitoring: The Future of Clinical Research
}

\author{
Madhuri Patel* \\ Texila American University, South America
}

Submission: September 20, 2017; Published: October 20, 2017

*Corresponding author: Madhuri Patel, Texila American University, Guyana, South America, Tel: 9558196458; Email:madhuri.patel1988@gmail.com

\section{Abstract}

The source data verification of clinical trial data is approach to linking quality of data and for being harmless side of regulatory. However, the regulations do not define the limit of Source data verification. This flexibility gives a chance to introduce innovation and application of various methods, targeted and remote monitoring, adaptive monitoring schedules and real time reporting. The remote site monitoring is one of these innovative methods to ensure the quality of data which can save time and cost, which can be utilize on more productive activities such as R\&D, implement new technology etc. And most importantly, it increases the work life balance of monitors.

However, remote monitoring involves verification of site documents from remote place. It has challenges as well. The source documents should be certified copies of electronic records, which might not be the case. Hence, remote monitoring cannot be totally replaced with on-site visit which can allow to see how site works. All trial should have delicate balance of remote and on-site monitoring throughout the study.

In this article, we will discuss potential opportunities and challenges of remote site monitoring along with potential solutions to overcome challenges. I am utilizing "Remote Monitoring" term to means a remote evaluation of clinical data at a location other than investigational site.

\section{Introduction}

The Monitoring has been always a considered a vital activity in order to conduct clinical trial and to ensure quality of data and procedures followed for particular protocol. First, I would like to mention about what remote monitoring us NOT. Very often, remote monitoring is confused with Risk-Based Monitoring, Remote monitoring allows monitoring activities which are previously conducted on-site, increase efficiency and speeding up time to market. CRA plays very crucial role, but without spending time onsite or travelling. Instead the CRA performs all the study monitoring processes remotely, using a secure online platform.

\section{Let Us Start with the Definition of Monitoring in ICH E6 (Good Clinical Practice) [1]}

"The act of overseeing the progress of a clinical trial, and of ensuring that it is conducted, recorded, and reported in accordance with the protocol, Standard Operating Procedures (SOPs), Good Clinical Practice (GCP), and the applicable regulatory requirement(s)." Section 5.18.3 of Guideline E6 states: "The sponsor should ensure that trials are adequately monitored. The sponsor should determine the appropriate extent and nature of monitoring. The determination of the extent and nature of monitoring should be based on considerations such as the objective, purpose, design, complexity, blinding, size and endpoints of the trial. In general there is a need for on-site monitoring, before, during, and after the trial; however, in exceptional circumstances, the sponsor may determine that central monitoring in conjunction with procedures such as investigators' training and meetings, and extensive written guidance can assure appropriate conduct of the trial in accordance with GCP. Statistically controlled sampling may be an acceptable method for selecting the data to be verified." So, the next question is why industry still wants to focus on site monitoring for $100 \%$ source data verification for most of trials?

\section{Discussion}

It has been always believed that more source data verification assures better quality data. Especially when regulatory does not define how much source data should be verified, sponsor and monitors are on the side of caution. In fact, FDA recommends a review of a "representative" number of subject records and not all records [2]. In August 2011, Monitoring Guidance Document (1988) is replaced to adopt Risk-Based Approach to Monitoring (Draft) $[3,4]$. To removing the stigma that $100 \%$ source data verification is the only way to product approval, FDA is encouraging sponsors to take credit for activities that 
are conducted throughout the study that contribute to the responsibility of monitoring.

It has been determined that the majority of finding which can be identified during on-site monitoring can be identifies during remote monitoring too. It also allows sponsors to meet regulatory obligations, while taking benefit of cost effectiveness and time saving. To conduct $100 \%$ source data verification, every site is treated the same, without special consideration for how many queries were issues or how many findings were recorded on previous on-site monitoring. However, it may not be the most cost effective or efficient. The purpose of clinical trial monitoring is to avoid errors that may compromise subject safety and well-being along with trial result [5]. The remote monitoring has a potential to significantly reduce resources required to meet basic requirements. It allows more frequent source data verification which increase the data integrity and allows to take mitigation action sooner than on-site monitoring. For example, with remote SDV, the study sponsor has the ability to more frequently evaluate patient safety events, identify early safety signals, and check protocol compliance issues such as inclusion/ exclusion compliance for subject recruitment, correct dosing of study medications through drug accountability monitoring and appropriate timing and response to safety laboratory blood testing [6].

Remote monitoring has been challenging to date because of the accessible infrastructure compliant to HIPAA and 21 CFR Part 11 which allows access to patient data, trial master file and regulatory documents.

Remote monitoring concept is future of clinical research, and we are not fully convinced to adopt this concept. We must make several practical consideration about therapeutic area phase and complexity of trial etc., and clearly this carries the risk of missing critical issues. Technological considerations related to data, hardware, and connectivity as well as expertise in implementing and integrating remote clinical trial components are also essential for the success of this concept. Although it may be expected to have remote monitoring method cost effective, initial implementation will likely be more expensive than traditional approach. Because, it required require parallel processes in place. The air of resistance toward this concept from clinical research teams and clinical research sites must be met with a gradual culture change to propel clinical trial conduct in the next wave of innovation and better serve the patient of the future. The digitization of clinical research through the remote trial concept is an essential step in that trend.

\section{Conclusion}

Instead of making frequent periodic on-site monitoring visit, it can be performed at flexible interval based on each site's performance and risk level as the study progresses. Sites deemed low-risk less often and Sites deemed high-risk would be visited more often. Although the remote monitoring may reduce shortterm costs may prove to have long term consequences. Hence, remote or on-site monitoring is not to mutually exclusive, but should be combined with overall review process and excellent communication throughout will result in high quality data and securing compliance. In turn, balanced combination approach can give confidence that that patients enrolled in their trials have been protected, their data is accurate.

Amazingly, some sponsors and sponsor representatives are still embracing the On-Site monitoring process and focusing on what could go wrong rather than the positives and understanding the intent of the FDA Guidance. I am hopeful that we will all take that critical step forward and embrace the change that is on our door step.

\section{References}

1. Rockville MD (1996) FDA: Food and Drug Administration, ICH E6 Good Clinical Practice Consolidated Guidance.

2. Getz KA (2011) Low hanging fruit in the fight against inefficiency. Applied Clinical Trials 20: 30-32.

3. US Department of Health and Human Services, Food and Drug Administration (2011) Guidance for Industry: oversight of clinical investigations-a risk-based approach to monitoring (DRAFT).

4. US Department of Health and Human Services, Food and Drug Administration (1988) Guidance for industry: guideline for the monitoring of clinical investigations.

5. Baigent C, Harrell FE, Buyse M, Emberson JR, Altman DG (2008) Ensuring trial validity by data quality assurance and diversification of monitoring methods. Clinical Trials 5(1): 49-55.

6. Stafford PB, Garrett A (2011) Using real-time data to drive better decisions, faster. Therapeutic Innovation \& Regulatory Science 45: 495-502. 
(C) This work is licensed under Creative Commons Attribution 4.0 License
Your next submission with Juniper Publishers will reach you the below assets

- Quality Editorial service

- Swift Peer Review

- Reprints availability

- E-prints Service

- Manuscript Podcast for convenient understanding

- Global attainment for your research

- Manuscript accessibility in different formats

( Pdf, E-pub, Full Text, Audio)

- Unceasing customer service

Track the below URL for one-step submission https://juniperpublishers.com/online-submission.php 